\title{
ENLARGEMENTS OF ALMOST OPEN MAPPINGS
}

\author{
ROBIN HARTE AND MARTIN MATHIEU ${ }^{1}$
}

\begin{abstract}
If the enlargement of a bounded linear operator has dense range, then the operator must be almost open.
\end{abstract}

Introduction. If $X$ is a normed space we write [1]

$$
\mathbf{Q}(X)=l_{\infty}(X) / c_{0}(X)
$$

for the enlargement of $X$, and if $T \in \mathrm{BL}(X, Y)$ is a bounded linear operator between normed spaces we write

$$
\mathbf{Q}(T): \mathbf{Q}(X) \rightarrow \mathbf{Q}(Y)
$$

for the operator induced by $T$, so that for each $x \in l_{\infty}(X)$ we have

$$
\mathbf{Q}(T)\left(x+c_{0}(X)\right)=(T x)+c_{0}(Y) \text {. }
$$

Now we recall that

$$
\mathbf{Q}(T) \text { one-one } \Rightarrow T \text { bounded below } \Rightarrow \mathbf{Q}(T) \text { bounded below }
$$
and [1, Theorem 4.1],

$$
\mathbf{Q}(T) \text { almost open } \Rightarrow \text { T almost open } \Rightarrow \mathbf{Q}(T) \text { open } .
$$

It is the purpose of this note to improve $(0.5)$ by confirming the conjecture (4.1.3) of [1].

\section{1}

TheOREM. If $T \in \mathrm{BL}(X, Y)$ is a bounded linear operator between normed spaces then

$$
\mathbf{Q}(T) \text { dense } \Rightarrow \text { T almost open } \Rightarrow \mathbf{Q}(T) \text { open } .
$$

Proof. Suppose $\varphi: l_{\infty} \rightarrow \mathbb{C}$ is a bounded linear functional for which

$$
c_{0} \subseteq \varphi^{-1}(0)
$$

then for each normed space $X$ we may define

$$
\hat{\varphi_{X}}: \mathbf{Q}\left(X^{\dagger}\right) \rightarrow \mathbf{Q}(X)^{\dagger}
$$

by setting, for each $f \in l_{\infty}\left(X^{\dagger}\right)$ and each $x \in l_{\infty}(X)$,

$$
\hat{\varphi_{X}}([f])([x])=\varphi\left(f_{0}\left(x_{.}\right)\right)
$$

here $X^{\dagger}$ denotes the usual dual of the normed space $X,[x]=x+c_{0}(X)$ and $[f]=f+c_{0}\left(X^{\dagger}\right)$ are cosets, and $f_{.}\left(x_{0}\right)=a \in l_{\infty}$ where $a_{n}=f_{n}\left(x_{n}\right)$ for each $n \in \mathbb{N}$. The reader should check, using (1.1.2), that the right-hand side of (1.1.4) depends

\footnotetext{
Received by the editors January 2, 1985 and, in revised form, February 8, 1985. 1980 Mathematics Subject Classification. Primary 47A99; Secondary 46B99.

${ }^{1}$ The second author gratefully acknowledges the support of the Studienstiftung des deutschen Volkes which made possible his visit to the Republic of Ireland.
} 
only on the cosets $[f]$ and $[x]$ and that the linear mapping $\hat{\varphi_{X}}([f]): \mathbf{Q}(X)^{\dagger} \rightarrow \mathbb{C}$ is bounded. Using the Hahn-Banach theorem we claim

$$
[0] \neq[f] \in \mathbf{Q}\left(X^{\dagger}\right) \Rightarrow \varphi\left(f_{.}\left(x_{.}\right)\right) \neq 0 \text { for some } x \in l_{\infty}(X), \varphi \in\left(l_{\infty} / c_{0}\right)^{\dagger} ;
$$

for if $f \in l_{\infty}\left(X^{\dagger}\right)$ is not in $c_{0}\left(X^{\dagger}\right)$, then $\limsup _{n}\left\|f_{n}\right\|>0$ and hence there is $x \in l_{\infty}(X)$ for which $\lim \sup _{n}\left|f_{n}\left(x_{n}\right)\right|>0$, so that $f_{.}\left(x_{.}\right) \in l_{\infty}$ is not in $c_{0}$. Now by the Hahn-Banach theorem there is $\varphi \in\left(l_{\infty}\right)^{\dagger}$ satisfying (1.1.2) for which $\varphi\left(f_{.}\left(x_{.}\right)\right) \neq$ 0 .

If $T \in \mathrm{BL}(X, Y)$ and $\varphi \in\left(l_{\infty}\right)^{\dagger}$ satisfies (1.1.2) then we claim

$$
\mathbf{Q}(T)^{\dagger} \circ \hat{\varphi_{Y}}=\hat{\varphi_{X}} \circ \mathbf{Q}\left(T^{\dagger}\right) ;
$$

for this is just the associative property

$$
\varphi(g .(T x) .)=\varphi((g T) .(x .)) \text { for each } x \in l_{\infty}(X), g \in l_{\infty}\left(Y^{\dagger}\right) .
$$

We are ready to make our final claim: if $T \in \operatorname{BL}(X, Y)$ then

$$
\mathbf{Q}(T)^{\dagger} \text { one-one } \Rightarrow \mathbf{Q}\left(T^{\dagger}\right) \text { one-one. }
$$

Indeed suppose $\mathbf{Q}\left(T^{\dagger}\right)$ is not one-one, so that there is $g \in l_{\infty}\left(Y^{\dagger}\right)$ for which

$$
g T \in c_{0}\left(X^{\dagger}\right) \text { and } g \notin c_{0}\left(Y^{\dagger}\right),
$$

and then by (1.1.5) there is $\varphi \in\left(l_{\infty}\right)^{\dagger}$ and $y \in l_{\infty}(Y)$ for which

$$
c_{0} \subseteq \varphi^{-1}(0) \text { and } \varphi\left(g_{.}\left(y_{.}\right)\right) \neq 0 \text {; }
$$

but now

$$
\mathbf{Q}(T)^{\dagger}\left(\varphi_{Y}^{\hat{Y}}[g]\right)=[0] \in \mathbf{Q}(X)^{\dagger} \text { and }[0] \neq \varphi_{Y}[g] \in \mathbf{Q}(Y)^{\dagger} \text {. }
$$

A familiar application of the Hahn-Banach separation theorem now gives (1.1.1): If $T \in \mathrm{BL}(X, Y)$ then

$$
\mathbf{Q}(T) \text { dense } \Rightarrow \mathbf{Q}(T)^{\dagger} \text { one-one } \Rightarrow \mathbf{Q}\left(T^{\dagger}\right) \text { one-one }
$$

and

$$
\mathbf{Q}\left(T^{\dagger}\right) \text { one-one } \Rightarrow T^{\dagger} \text { bounded below } \Rightarrow T \text { almost open } .
$$

For an alternative proof of Theorem 1.1 we can use ultrafilters on $\mathbb{N}$ instead of linear functionals on $l_{\infty} / c_{0}[2]$.

\section{REFERENCES}

1. R. E. Harte, Almost open mappings between normed spaces, Proc. Amer. Math. Soc. 90 (1984), 243-249.

2. M. Mathieu, On approximate point spectra of elementary operators, Semesterbericht functionalanalysis Tubingen, 1985.

Department of Mathematics, University College, Cork, Republic of Ireland

Mathematisches Institut, TÚbingen, Federal Republic of Germany (Current address of Martin Mathieu)

Current address (Robin Harte): Division of Mathematical Sciences, University of Iowa, Iowa City, Iowa 52242 